\title{
Relación entre Capacidad de Innovación e Índice de Innovación en América Latina
}

\author{
Isabel Cristina Quintero Sepúlveda ${ }^{1}$, Yovanny Ospina Nieto ${ }^{2}$, Darío J. Quiroga Parra ${ }^{3}$, \\ Rolando-Arturo Cubillos-González ${ }^{4}$
}

Resumen: Según el Manual de Oslo, las capacidades de innovación se relacionan con las capacidades generales de la empresa y se consideran un conjunto de activos valiosos que la empresa utiliza para establecer estrategias de innovación. Tienen relación directa con la ventaja competitiva y el crecimiento de las ventas.

A nivel latinoamericano, ha sido común el uso de indicadores internacionales para medir la capacidad de innovación de las empresas, no obstante, estos conceptos no muestran igual claridad académica en el estado del arte de América Latina. Este artículo tiene por objetivo explicar la relación que se da entre la capacidad de innovación y el índice de innovación en América Latina con respecto al contexto internacional. Para ello se identificó en primer lugar los países que evidencian estudios empíricos relacionados con la capacidad de innovación. Y, en segundo lugar, se estableció la relación existente entre la capacidad de innovación y el índice de innovación. Como resultado se propuso un índice de eficiencia técnica para analizar las diferencias que se dan entre los países de América Latina en relación con el contexto internacional. El principal aporte del estudio es que logra explicar las capacidades de innovación en AL en relación con los resultados de innovación evidenciados en el índice global de innovación mediante el índice de eficiencia técnica propuesto. Los resultados indican que los índices de eficiencia técnica son similares en los dos grupos de países analizados, y a mayor capacidad innovadora, mayor índice de innovación.

Palabras clave: Capacidades de innovación; índice de innovación; eficiencia técnica; Latinoamérica

\begin{abstract}
The Oslo Manual said that it related innovation capabilities to the general abilities of the company. Also, it set of valuable assets that the company uses to establish innovation strategies. It related it to them to competitive advantage and growth in sales. Using international indicators to measure the innovation capacity of companies has been usual at the Latin American level. However, these concepts do not show the same academic clarity in the state-of-the-art. This article aims to explain the relationship between innovation capacity and the innovation index in Latin America regarding the international context. The method comprised a systematic review identifying first the countries that evidence empirical studies related to innovation capacity. And second, it established that the relationship between the innovation capacity and the innovation index. Result proposed a technical efficiency index to analyse the differences between Latin America and the international context. The principal contribution of the study is that it explains the innovation capacities of global innovation through the proposed technical efficiency index. The results show that the technical efficiency indices are similar in the two groups of countries analysed, and the higher the innovative capacity, the higher the innovation index.
\end{abstract}

Keywords: Innovation capabilities; innovation index; technical efficiency; Latin America

Submitted: September $15^{\text {th }}, 2021 /$ Approved: October $26^{\text {th }}, 2021$

\section{Introducción}

De acuerdo con el Manual de Oslo (OCDE \& Eurostat, 2018), las capacidades de innovación están estrechamente relacionadas con las capacidades generales de la organización, y abarcan todas las actividades para iniciar, desarrollar y lograr resultados de innovación. Son un activo valioso para generar ventajas competitivas y para la implementación estratégica; es la base de los procesos principales de la empresa. Están presentes en las diferentes prácticas organizativas, como el desarrollo de nuevos productos, la incorporación de nuevas tecnologías, la generación y adopción de procesos para satisfacer necesidades futuras y responder adecuadamente a los cambios del entorno y la competencia (Rajapathirana \& Hui, 2018).
La relación de las capacidades de innovación con los resultados de innovación muestra estar vigentes en la literatura internacional. A nivel latinoamericano $(\mathrm{AL})$ ha sido común el uso de indicadores internacionales para medir la capacidad de innovación de las empresas en diversos estudios empíricos, no obstante, estos conceptos no muestran igual claridad académica en el estado del arte de AL.

La relación de las capacidades de innovación con los resultados de innovación muestra estar vigentes en la literatura internacional. A nivel latinoamericano $(\mathrm{AL})$ ha sido común el uso de indicadores internacionales para medir la capacidad de innovación de las empresas en diversos estudios empíricos, no obstante, estos conceptos no muestran igual claridad académica en el estado del arte de AL.

(1) Facultad de Administración, Universidad Pontificia Bolivariana, Palmira, Colombia

(2) Facultad de Psicología, Universidad Pontificia Bolivariana, Palmira, Colombia

(3)Facultad de Administración, Economía y Contaduría, Universidad Cooperativa de Colombia, Cali, Colombia

(4) Facultad de Ingeniería, Universidad Pontificia Bolivariana, Medellín, Colombia

${ }^{*}$ Corresponding author: isabel.quintero@upb.edu.co 
Los avances en innovación y en capacidades de innovación de acuerdo con los resultados del índice global de innovación (Cornell University et al., 2019; WIPO, 2019) y del informe de competitividad (Schwab, 2019) siguen evidenciando los rezagos de los países latinoamericanos en temas de innovación y capacidades de innovación con respecto al contexto internacional. Entonces cabe preguntarse ¿qué relación se da entre la capacidad de innovación y el índice de innovación en América Latina con respecto al contexto internacional?Para dar respuesta a la pregunta planteada, este estudio tiene por objetivo explicar la relación que se da entre la capacidad de innovación y el índice de innovación en América Latina $(\mathrm{AL})$ con respecto al contexto internacional. Para lo cual se identificó en primer lugar los países que evidencian estudios empíricos relacionados con la capacidad de innovación. En segundo lugar, se estableció la relación existente entre la capacidad de innovación y el índice de innovación, para finalmente analizar las diferencias que se dan entre los países de América Latina en relación con el contexto internacional.

Para dar respuesta a la pregunta planteada, este estudio tiene por objetivo explicar la relación que se da entre la capacidad de innovación y el índice de innovación en América Latina (AL) con respecto al contexto internacional. Para lo cual se identificó en primer lugar los países que evidencian estudios empíricos relacionados con la capacidad de innovación. En segundo lugar, se estableció la relación existente entre la capacidad de innovación y el índice de innovación, para finalmente analizar las diferencias que se dan entre los países de América Latina en relación con el contexto internacional.

Las metodologías utilizadas fueron: 1) Uso del método PRISMA (Moher et al., 2009) para revisión literaria. 2) Se hizo un análisis de correlación para establecer el vínculo existente entre la capacidad de innovación y el índice de innovación. Se validó a través de tres estadísticos, que fueron el Alfa de Crombach, el coeficiente de correlación de Pearson, y su respectivo valor de significancia. 3) Se propuso un índice de eficiencia técnica de la capacidad de innovación con relación al índice innovación para explicar las diferencias que se dan entre los países de AL y el contexto internacional (Dosi, 1992; Teece, 2014).

Los resultados evidencian que en AL los países que más estudios han realizado sobre capacidades de innovación son Brasil, México y Colombia, y en menor proporción Chile, Ecuador y Perú. En cuanto a las variables analizadas, se encontró una correlación positiva entre capacidad de innovación e índice de innovación que se da para los países de AL que son analizados tanto por el Informe Global de Innovación como por el Informe Global de Competitividad. Al aplicar el índice de eficiencia técnica comparando los principales países del ranking de innovación y de competitividad global en comparación con los países de AL, se encontró que ambos grupos de países tienen resultados similares, y las diferencias entre los países se dan por el nivel de capacidad de innovación con una relación directa con los resultados del índice de innovación, de tal manera que a mayor nivel de capacidad de innovación, mayor índice de innovación tienen los países analizados.

\section{Revisión de literatura}

\section{Capacidades de innovación}

La literatura internacional, marca una fuerte tendencia hacia el enfoque de las capacidades de innovación, tecnológicas, y tecnológicas de innovación. Las capacidades tecnológicas y tecnológicas de innovación se consideran un conjunto de activos y recursos que la empresa utiliza para establecer estrategias de innovación (Guan \& Ma, 2003; Yam et al., 2004). Ellas involucran la tecnología, el producto, los procesos, el conocimiento, la experiencia y la organización y tienen una relación directa con la ventaja competitiva y el crecimiento de las ventas.

Las capacidades de innovación son consideradas como la aptitud de la empresa para organizarse y lograr resultados de innovación (Robledo Velasquez, 2016). Estas son un activo especial, tácito, y relacionado con la experiencia y la experimentación para el desarrollo y comercialización, tanto de nuevas tecnologías y productos, como la adopción de nuevos procesos, especialmente en contextos de rápido cambio tecnológico (Guan \& Ma, 2003). Esta está presente en las diferentes prácticas organizativas, como el desarrollo de nuevos productos, la incorporación de nuevas tecnologías a los productos, la generación y adopción de procesos para satisfacer necesidades futuras y responder adecuadamente a los cambios del entorno y la competencia (Rajapathirana \& Hui, 2018).

$\mathrm{Al}$ articular el concepto de capacidad dinámica con las capacidades de innovación, Cheng \& Chen (2013) plantean que las capacidades de innovación dinámica son capacidades operativas que incluyen procesos de aprendizaje organizacional. Así como rutinas fundamentadas en el conocimiento de la innovación. Son consideradas como aquellas que son difíciles de transferir y de imitar, que las empresas utilizan para desarrollar, integrar y reconfigurar los recursos existentes y nuevos así como las capacidades operativas.

\section{Las capacidades de innovación en AL}

La literatura sobre las capacidades de innovación en AL, evidencia que ésta establece sus bases teóricas en postulados internacionales, Sin embargo, no se observa una teoría específica ni una escuela explícita que facilite comprender la perspectiva desde la cual se está abordando el tema. También se identifica una tendencia hacia los estudios sobre capacidades dinámicas y capacidades de innovación, éstas últimas prevalecen en los estudios de Brasil. En general, se encontró un consenso de la incidencia de las capacidades de innovación respecto a los resultados de innovación en las empresas y su efecto en el desempeño.

En la literatura consultada los estudios toman diversos enfoques. La mayoría de éstos son de corte transversal y reconocen la importancia de profundizar el tema de manera longitudinal. Los estudios de caso se enfocan en tratar de explicar cómo es el desarrollo de la innovación tomando como base la teoría de las capacidades dinámicas y/o de innovación principalmente en Brasil y México. De su parte, los estudios cuantitativos buscan identificar capacidades de innovación y capacidades dinámicas relevantes en los sectores específicos, donde se realizan las investigaciones con el objetivo de aportar elementos que promuevan el mejoramiento de la competitividad. 
En los estudios que analizan las capacidades dinámicas en AL aún no hay un consenso frente a las variables que explican este tipo de capacidades, así mismo los propósitos se enfocan en su influencia sobre aspectos la resiliencia y la sostenibilidad, las capacidades humanas, organizativas y tecnológicas (Almeida Souza et al., 2017); la exploración y explotación del mercado, la capacidad de aprendizaje y adaptación (Gonçalves Tondolo et al., 2010); el fortalecimiento de relaciones comerciales internacionales (Enjolras et al., 2020; Griffith \& Harvey, 2001); la cultura empresarial emprendedora y el conocimiento del entorno (Herrmann et al., 2017); la sostenibilidad y la industria 4.0 (Liboni et al., 2018), el capital humano, relacional y organizacional (Daou et al., 2013).

De otra parte, los estudios sobre capacidades de innovación, se relacionan con otras variables que la conforman (Barbosa Ferreira et al., 2019; Cevallos et al., 2018; Jardon, 2018; Lema et al., 2015; López Montoya \& Acosta-Prado, 2019; Martínez Marín \& Arango Aramburo, 2017; Narcizo et al., 2013; Wu, 2019), y desde diversos marcos de análisis: nivel de clúster (Becerra Rodríguez, F.; Valencia Naranjo, 2008; Bittencourt et al., 2019; Díaz Pérez et al., 2009; Mejía Vallejo \& Arias-Pérez, 2017; Zartha Sossa et al., 2016), nivel de empresa (Figueiredo, 2014; LópezCabarcos et al., 2015; López Montoya \& Acosta-Prado, 2019; Maldonado-Guzmán et al., 2019; Novick et al., 2013; Oliveira et al., 2019; Quintero S. et al., 2019; Ramirez et al., 2019; Tarraço et al., 2019); y proceso de internacionalización (Fleury et al., 2013).También se encontraron estudios en AL que validan instrumentos para evaluar las capacidades dinámicas (Cabrera-Moya, 2017), así como de capacidades de innovación (Cevallos et al., 2018; López M. et al., 2018; Serrano García \& Robledo Velásquez, 2013; Zartha Sossa et al., 2016).

También se encontraron estudios en AL que validan instrumentos para evaluar las capacidades dinámicas (Cabrera-Moya, 2017), así como de capacidades de innovación (Cevallos et al., 2018; López M. et al., 2018; Serrano García \& Robledo Velásquez, 2013; Zartha Sossa et al., 2016).

No obstante, la clara relación de las capacidades dinámicas de innovación con: estrategia, productividad, ventaja competitiva, competitividad de las empresas y las capacidades basadas en recursos muestran estar vigentes en la literatura internacional, estos conceptos no muestran igual claridad académica en el estado del arte de AL. Es evidente que, el concepto de D.J. Teece et al., (1990) sobre las capacidades dinámicas, esto es, unas capacidades fundamentadas en los mecanismos de aprendizaje y acumulación de conocimiento como elementos intangibles al interior de las empresas, no es muy evidente en la región.

Se deduce que, en AL existe bajos niveles de uso de la información y de absorción de conocimiento aun; dados los dilatados procesos de uso intensivo de las tecnologías de la información y la comunicación. De hecho, no se observa en AL una real integración que facilite la reconfiguración de las habilidades internas y externas organizacionales. Elementos como el análisis de entorno cambiante no muestra ser un factor de prioridad. De manera que, a la adaptación al entorno para integrar y reconfigurar recursos, habilidades y competencias internas y externas, que faciliten impactarlo, son una necesidad empresarial de AL.

\section{Los índices de innovación}

Para desarrollar esta investigación se tomó como base el ranking de innovación (Cornell University et al., 2019) que presenta la clasificación mundial de los países de acuerdo con la generación de innovación a partir de la medición de 7 aspectos: instituciones, capital humano e investigación, infraestructura, desarrollo de mercados, desarrollo empresarial, producción de conocimiento y tecnología, y creatividad.

También se analizó el índice mundial de competitividad (World Economic Forum, 2018) que presenta el ranking de los países más competitivos basándose en 12 pilares: instituciones, infraestructura, adopción de CTI, estabilidad macroeconómica, salud, habilidades de la fuerza laboral, mercado de productos, mercado laboral, sistema financiero, tamaño del mercado, dinámica empresarial, y capacidades de innovación.

Si bien, algunos indicadores son comunes para ambos rankings, se evidencia la importancia que tienen las capacidades de innovación sobre los resultados de innovación y el desempeño empresarial (Barbosa Ferreira et al., 2019; Cabrera-Moya, 2017; Díaz Pérez et al., 2009; Narcizo et al., 2013; Rodriguez et al., 2013; Souza et al., 2017; Teece, 2017; Valdez-Juárez et al., 2018) . Pero no se evidencian estudios que profundicen frente a la relación entre la capacidad de innovación y el índice de innovación en los países de AL.

\section{Metodología}

Esta investigación se realizó con un método combinado. Para identificar los países que estudian las capacidades de innovación se hizo una revisión sistemática con el método PRISMA (Moher et al., 2009), que se enfoca en un informe de revisiones que evalúan ensayos aleatorios en este caso identificados en la base de datos Scopus. Luego, se analizaron los datos con el software VOSviewer, utilizado para construir redes de términos obtenidos de la literatura científica, e identificar redes bibliométricas con base en citación, relaciones bibliográficas y de coautoría.

La revisión fue útil para la contextualización del estado del estudio de las capacidades de innovación en América Latina y el planteamiento de la relación existente entre la capacidad de innovación y el índice de innovación tanto en Latinoamérica como en el contexto internacional de manera comparada. Se identificaron 145 documentos. La revisión se organizó en datos tanto estructurados como no estructurados. La información estructurada obtenida de la base de datos Scopus se hizo en una línea de tiempo de 2014 a 2020 y se acotó a temas de economía y administración para identificar las investigaciones y analizarlas por diferentes parámetros.

Para establecer la relación entre la capacidad de innovación y los índices de innovación, se tuvo en cuenta, además de la literatura científica, tanto el informe global de innovación 2019 (WIPO, 2019) como el Índice global de competitividad (World Economic Forum, 2019). Se analizaron los resultados de los 19 países de AL con relación al índice 
de innovación general, así como el índice de capacidad de innovación, que constituye uno de los 12 pilares que se tienen en cuenta para determinar el índice de competitividad de cada país. Así mismo se hizo proporcionalmente una comparación con los países con mejores índices en estos informes (20 países).

El análisis de la relación entre la capacidad de innovación y el índice de innovación se realizó un análisis correlacional entre las dos variables. Los resultados indican una correlación positiva entre éstas y un nivel de significancia positivo.

Una vez confirmada la correlación positiva entre la capacidad de innovación y el índice de innovación, se estableció el índice de eficiencia técnica para tratar de explicar las diferencias en los resultados que se han tenido en estas variables en los países latinoamericanos en comparación con los 20 primeros países del mundo, de acuerdo con los informes WIPO (2019) y WEF (2019).

\section{Resultados}

Como se presentó en el apartado metodológico, para explicar la relación que se da entre la capacidad de innovación y el índice de innovación en América Latina con respecto al contexto internacional, se hizo una revisión sistemática con tres momentos: 1) Identificación de los países que estudian las capacidades de innovación en AL. 2) Análisis de correlación para establecer el vínculo existente entre la capacidad de innovación y el índice de innovación utilizando tres estadísticos de validación, que fueron el Alfa de Crombach, el coeficiente de correlación de Pearson, y el valor de significancia. 3) Como resultado se propuso un índice de eficiencia técnica de la capacidad de innovación con relación al índice innovación para explicar las diferencias que se dan entre los países de AL y el contexto internacional.

\section{Países que estudian las capacidades de innovación}

Para identificar los países que estudian las capacidades de innovación, se hizo una revisión exhaustiva de los documentos y se identificaron 50 que analizan las capacidades de innovación en un país o empresa latinoamericana. El 63\% de los estudios son cuantitativos, el 25\% cualitativos y el $12 \%$ mixtos.

La Tabla 1 presenta las 10 palabras clave principales de acuerdo con la ocurrencia y con la fuerza de enlace. De las variables definidas en esta investigación, la primera palabra "innovation capabilities" apareció 21 veces y tuvo una fuerza de enlace total del 37\%. La segunda palabra de interés relacionada con los países de Latinoamérica, "Brazil” apareció 16 veces, y mostró una fuerza de enlace total del $21 \%$. "Latinamerica" apareció 12 veces con una fuerza de enlace del $7 \%$. "Colombia” apareció 9 veces con una fuerza de enlace del 7\%. Otras palabras relacionadas como "innovation" apareció 30 veces con una fuerza de enlace del 35\% y la palabra "Dynamic capabilities" apareció 26 veces con una fuerza de enlace del $23 \%$.
Tabla 1: Relación de palabras con las variables de estudio.

\begin{tabular}{lcc}
\hline Keyword & Occurrences & Total link strength \\
\hline Innovation & 30 & 35 \\
Dynamic capabilities & 26 & 23 \\
Innovation capabilities & 21 & 37 \\
Brazil & 16 & 21 \\
Latinamerica & 12 & 7 \\
Industrial management & 10 & 23 \\
Colombia & 9 & 7 \\
Competition & 7 & 15 \\
Knowledge management & 7 & 8 \\
Sustainable development & 7 & 20 \\
\hline
\end{tabular}

Nota: Elaboración propia con Software VOSViewer.

La Tabla 2 presenta los 13 países más importantes en la revisión de literatura. Se observa que los cinco primeros países respecto a la fuerza de enlace son España, Reino Unido, Brasil, Colombia y México. Los documentos indican que existe una prevalencia de estudios en tres países de AL: Brasil, Colombia y México.

Tabla 2: Relación de países con las variables de estudio.

\begin{tabular}{cccc}
\hline Country & Documents & Citations & Total link strength \\
\hline Spain & 15 & 29 & 14 \\
United Kingdom & 12 & 106 & 14 \\
Brazil & 28 & 270 & 9 \\
Colombia & 24 & 13 & 9 \\
Mexico & 16 & 49 & 8 \\
Italy & 3 & 22 & 6 \\
France & 5 & 14 & 5 \\
Netherlands & 3 & 2 & 5 \\
United States & 12 & 8 & 5 \\
Australia & 3 & 24 & 4 \\
Chile & 4 & 6 & 3 \\
Argentina & 3 & 1 & 2 \\
Perú & 4 & 1 &
\end{tabular}

Nota: Elaboración propia con Software VOSViewer.

El análisis de la relación entre las variables de estudio, evidencia que las investigaciones en AL se relacionan con "innovación”, "gestión del conocimiento" y "capacidades dinámicas". Los estudios de Colombia se relacionan con "capacidades de innovación", "desarrollo sostenible", "capacidades dinámicas" e "innovación". Se observa que los estudios de Brasil tienen mayor relación con la variable "capacidades de innovación" que el resto de los países de AL en donde prevalece la relación con "innovación” y "capacidades dinámicas". También se encontró una fuerte relación entre los países de América Latina y España, Reino Unido y USA principalmente. 
Posteriormente, se hizo una selección de los documentos teniendo en cuenta tres categorías. 1) Investigaciones que estudian las capacidades de innovación en los países de AL. 2) Estudios que evalúan capacidades de innovación y los índices de innovación. 3) Documentos que analizan la innovación en AL.

De los documentos analizados, el país con mayor participación en las investigaciones sobre las capacidades de innovación es Colombia (33\%). Brasil tiene mayor fuerza de enlace e impacto en las citaciones. Así mismo, en menor proporción, se encontraron estudios en Chile y Ecuador (4\% de participación cada uno) y Perú (2\%), así como estudios generales para AL (2\%).

También se seleccionaron los estudios que realizan evaluaciones respecto al nivel de desarrollo de las capacidades de innovación, la búsqueda arrojó 18 documentos, el $83 \%$ de ellos son cuantitativos y el $17 \%$ cualitativos. Estos estudios, fueron realizados para México y Brasil ( $25 \%$ en cada uno), seguido de estudios de carácter internacional, para AL y para Colombia ( $15 \%$ en cada uno), en menor proporción se encontraron estudios para Chile (5\%).

Con relación a los estudios generales sobre innovación en AL, se encontraron 30 documentos, el 53\% cualitativos, el 44\% cuantitativos y el $3 \%$ mixtos. Los países para los que se realizaron estas investigaciones fueron Brasil (32\%), Colombia (23\%), AL en general (19\%), estudios internacionales comparativos (13\%), y en menor proporción México (6\%) y Chile (3\%).

Relación entre la capacidad de innovación y el índice de innovación. La síntesis de los análisis de correlación entre las variables capacidad de innovación e índice de innovación que se da para los países de AL que son analizados tanto por el Informe Global de Innovación como por el Informe Global de Competitividad, que se presenta en la Tabla 3, indica los valores de las medias obtenidas con una desviación estándar alta, lo cual puede estar explicado por las diferencias de los resultados entre los países que obtuvieron los índices más altos en comparación con los de AL tal como se muestra en el gráfico de dispersión (ver Figura 1).

El valor de correlación para las variables latentes fue de 0,974. El nivel de significancia se encuentra en los parámetros adecuados $(\mathrm{p}<0.05)$. El valor del Alfa de Crombach $(0,987)$ basado en elementos estandarizados indica una alta fiabilidad (consistencia interna), tal como se presenta en la Tabla 3.

Tabla 3: Correlaciones, medias, desviaciones estándar y significancia

\begin{tabular}{lcccc}
\hline Variables & Media & $\begin{array}{c}\text { Desviación } \\
\text { estándar }\end{array}$ & $\mathbf{1}$ & $\mathbf{2}$ \\
\hline 1. Índice de innovación (Inn) & 44,1 & 14.5 & 0,974 & \\
2. Capacidad de innovación (IC) & 55,3 & 21 & 0,000 & 0,974 \\
\hline
\end{tabular}

Nota: $\mathrm{N}=39$.
Figura 1: Gráfico de dispersión

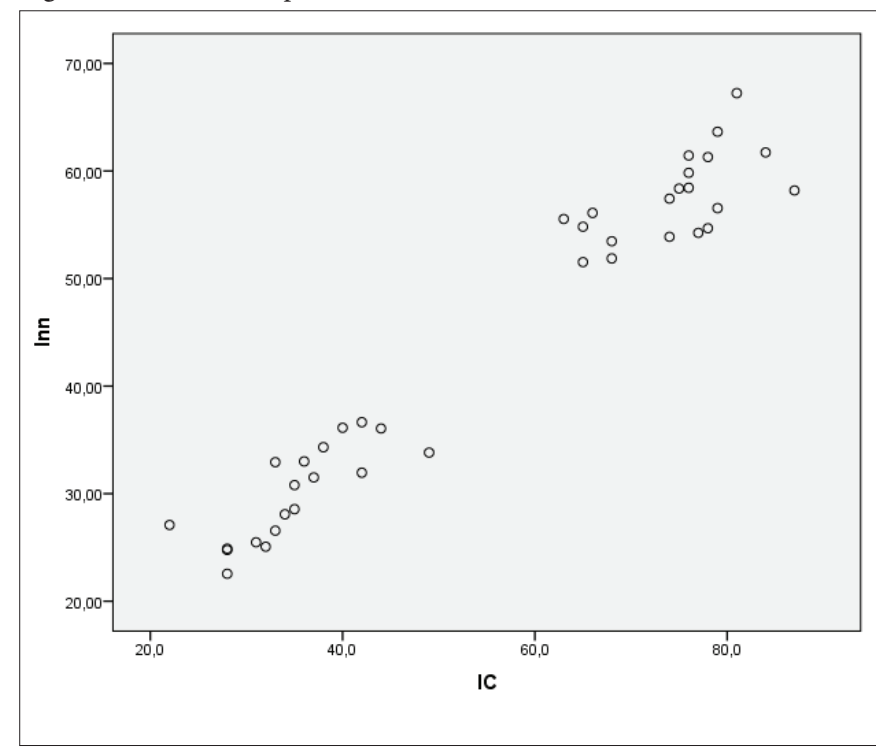

Nota: Elaboración con Software SPSS.

Los resultados indican una correlación positiva entre las variables analizadas. El gráfico de dispersión (Figura 1) muestra las diferencias en los resultados entre los países de AL (cuadrante inferior), frente a los países con mayores índices tanto de innovación como de capacidad de innovación (cuadrante superior).

Índice de eficiencia técnica que explica la brecha de la capacidad de innovación entre los países de América Latina y el contexto internacional.

Desde el punto de vista económico, la eficiencia técnica está relacionada con el desempeño en el uso de los recursos (Cachanosky, 2012; Fried, H.O.; Knox Lovell, C.A.; Smidt, 2008; González Fidalgo, 1999). En el análisis de la eficiencia en relación con variables como la innovación, o la eficiencia de los países, los estudios evidencian la pertinencia de del uso de los modelos de eficiencia (Delgado \& Álvarez, 2005; Farrell, 1957; Zamora Torres \& Tello, 2018).

Este análisis toma como base los índices de medida de eficiencia absoluta que no utilizan frontera (Peretto, 2017) y analiza los resultados obtenidos (índice de innovación) respecto a los factores o recursos empleados (capacidad de innovación). Esto permite lograr una mayor comprensión entre las diferencias que se dan entre los países de AL frente al contexto internacional, en relación con las capacidades de innovación y el índice de innovación que tienen estos países menos desarrollados.

Una vez se establece la correlación entre las 2 variables, se hizo un análisis de los resultados con base en la eficiencia técnica (Fried, H.O.; Knox Lovell, C.A.; Smidt, 2008; Peretto, 2017) de la capacidad de innovación respecto al índice de innovación $(\mathrm{E}=\mathrm{Inn} / \mathrm{IC})$ tanto para los países con mayor puntuación como para los países de AL. Los resultados como se indica en la Tabla 4, evidencian que estos países tienen un indicador de eficiencia técnica de la capacidad de 
innovación entre 0,67 y 0,88 . Por su parte para los países de AL, el indicador de eficiencia técnica de la capacidad de innovación con respecto al índice de innovación está entre 0,69 y 1,23 como se indica en la Tabla 5.

Tabla 4: Índice de eficiencia técnica de la capacidad de innovación calculado para los 20 países mejor clasificados según el índice global de innovación.

\begin{tabular}{|c|c|c|c|c|c|}
\hline \multirow{2}{*}{ País } & \multicolumn{2}{|c|}{ Índice de innovación } & \multicolumn{2}{|c|}{ Capacidad de Innovación } & \multirow{2}{*}{$\begin{array}{c}\text { Indicador de eficiencia } \\
\text { técnica }\end{array}$} \\
\hline & Posición & Puntaje & Posición & Puntaje & \\
\hline Switzerland & 1 & 67 & 5 & 81 & 0,830 \\
\hline Sweden & 2 & 64 & 8 & 79 & 0,806 \\
\hline USA & 3 & 62 & 2 & 84 & 0,735 \\
\hline Netherlands & 4 & 61 & 4 & 76 & 0,808 \\
\hline United Kindong & 5 & 61 & 9 & 78 & 0,786 \\
\hline Finland & 6 & 60 & 11 & 76 & 0,787 \\
\hline Denmark & 7 & 67 & 10 & 76 & 0,769 \\
\hline Singapore & 8 & 64 & 1 & 75 & 0,778 \\
\hline Germany & 9 & 62 & 7 & 87 & 0,669 \\
\hline Israel & 10 & 61 & 20 & 74 & 0,776 \\
\hline Republic of Korea & 11 & 61 & 13 & 79 & 0,716 \\
\hline Ireland & 12 & 60 & 24 & 66 & 0,850 \\
\hline Hong Kong, China & 13 & 58 & 3 & 63 & 0,882 \\
\hline China & 14 & 58 & 28 & 65 & 0,843 \\
\hline Japan & 15 & 58 & 6 & 78 & 0,701 \\
\hline France & 16 & 57 & 15 & 77 & 0,705 \\
\hline Canada & 17 & 57 & 14 & 74 & 0,728 \\
\hline Luxembourg & 18 & 56 & 18 & 68 & 0,786 \\
\hline Norway & 19 & 56 & 17 & 68 & 0,763 \\
\hline Iceland & 20 & 55 & 26 & 65 & 0,793 \\
\hline
\end{tabular}

Nota: Datos tomados del Índice Global de Innovación y el Informe Global de Competitividad, año 2019.

Tabla 5: Índice de eficiencia técnica de la capacidad de innovación calculado para los 19 países de AL que son evaluados por el índice global de innovación.

\begin{tabular}{|c|c|c|c|c|c|}
\hline \multirow{2}{*}{ País } & \multicolumn{2}{|c|}{ Î́ndice de innovación } & \multicolumn{2}{|c|}{ Capacidad de Innovación } & \multirow{2}{*}{$\begin{array}{c}\text { Indicador de eficien- } \\
\text { cia técnica }\end{array}$} \\
\hline & Posición & Puntaje & Posición & Puntaje & \\
\hline Chile & 51 & 37 & 33 & 42 & 0,87 \\
\hline Costa Rica & 55 & 36 & 62 & 40 & 0,90 \\
\hline México & 56 & 36 & 48 & 44 & 0,82 \\
\hline Uruguay & 62 & 34 & 54 & 38 & 0,90 \\
\hline Brasil & 66 & 34 & 71 & 49 & 0,69 \\
\hline Colombia & 67 & 33 & 57 & 36 & 0,92 \\
\hline Perú & 69 & 33 & 65 & 33 & 1,00 \\
\hline Argentina & 73 & 32 & 83 & 42 & 0,76 \\
\hline Panamá & 75 & 32 & 66 & 37 & 0,85 \\
\hline Jamaica & 81 & 31 & 80 & 35 & 0,88 \\
\hline República Dominicana & 87 & 29 & 78 & 35 & 0,82 \\
\hline Trinidad and Tobago & 91 & 28 & 79 & 34 & 0,83 \\
\hline Paraguay & 95 & 27 & 97 & 22 & 1,23 \\
\hline Ecuador & 99 & 27 & 90 & 33 & 0,80 \\
\hline Honduras & 104 & 25 & 101 & 31 & 0,82 \\
\hline Guatemala & 107 & 25 & 98 & 32 & 0,78 \\
\hline El Salvador & 108 & 25 & 103 & 28 & 0,89 \\
\hline Bolivia & 110 & 25 & 107 & 28 & 0,88 \\
\hline Nicaragua & 120 & 23 & 109 & 28 & 0,81 \\
\hline
\end{tabular}

Nota: Elaboración propia. 
Como se observa en la Tabla 4 y en la Tabla 5, los 20 primeros países del mundo tienen un índice de innovación entre 55 y 67 puntos y la capacidad de innovación entre 63 y 87 puntos; mientras que los países de AL tienen un índice de innovación entre 23 y 37 puntos y la capacidad de innovación entre 22 y 49 puntos. Al compararse estos resultados con el índice de eficiencia técnica calculado, se observa que los dos grupos de países analizados tienen un resultado similar. Por lo tanto, las diferencias entre los países se dan por el nivel de capacidad de innovación con una relación directa con los resultados del índice de innovación.

\section{Conclusiones}

La literatura consultada identificó una prevalencia de los estudios en Brasil, México y Colombia. Esta se ha venido desarrollando desde finales de 1998 de manera intermitente. De esta forma la innovación como capacidad en algunos contextos ha sido asumida como una estrategia para lograr ganancias de productividad y mejorar la competitividad internacional de las empresas y productos. Por el contrario, también ésta ha sido el camino para favorecer un mejor nivel de vida, además de fortalecer y potenciar el funcionamiento de las instituciones.

A pesar de la diversidad de estudios empíricos enfocados principalmente a: primero, contrastar los postulados teóricos internacionales en $\mathrm{AL}$; segundo, identificar aspectos propios del contexto que inciden en la actividad innovadora; y tercero, identificar los sectores en los que se realizan esfuerzos respecto a las capacidades de innovación de las empresas; hay una escasa evidencia de estudios que evalúen las capacidades de innovación en el contexto empresarial de AL, aspecto que dificulta identificar tanto oportunidades como brechas que deben superar las empresas con miras a fortalecer su actividad innovadora como pilar de la competitividad.

A pesar de que algunos de los indicadores de capacidades de innovación al interior de la empresa están presentes en la medición de la capacidad de innovación que utiliza el Foro Económico Mundial para medir esta variable en los países, hay una dificultad en la comparabilidad de los resultados a nivel micro, al no existir suficiente claridad en los indicadores macro, es decir que los resultados aún están generalizados y no por sectores y empresas.

El uso de indicadores internacionales para medir la capacidad de innovación de las empresas ha sido utilizado en diversos estudios empíricos a nivel latinoamericano como el de Dutrénit, Natera, Puchet Anyul, \& Vera-Cruz (2019) quienes evidencian las restricciones que tienen las métricas actuales para medir la acumulación de capacidades tecnológicas en países latinoamericanos, las cuales han sido adaptadas de los países desarrollados.

A nivel latinoamericano, la identificación de capacidades de innovación también se realiza mediante parámetros e instrumentos de otros países. Sin embargo no se evidencia un análisis de la pertinencia de los indicadores para el contexto y son adoptados bajo las posturas filosóficas europeas y americanas, demostrando la falta de análisis epistemológico respecto a la concepción de la innovación y las capacidades de innovación en las empresas de AL.
El análisis de la literatura internacional de las capacidades dinámicas presenta importantes avances y consolidación de conceptos académicos; sin embargo los avances en innovación y en capacidades de innovación de acuerdo con los resultados del índice global de innovación (Cornell University, INSEAD, \& WIPO, 2019) y del informe de competitividad (Schwab, 2019) siguen evidenciando los rezagos de los países de AL.

La relación entre las variables que conforman el índice de innovación y las capacidades de innovación se observa como una prioridad de estudio y profundidad académica tanto desde el contexto general en AL, como empresarial para contribuir a la consolidación de la competitividad en el contexto global. Generándose aquí una línea de investigación.

El valor agregado del presente estudio y aporte a la literatura es que logra explicar las capacidades de innovación en AL en relación con los resultados de innovación evidenciados en el índice global de innovación mediante el índice de eficiencia técnica propuesto. Los resultados indican que los índices de eficiencia técnica son similares en los dos grupos de países analizados, y a mayor capacidad innovadora, mayor índice de innovación.

\section{Referencias}

Barbosa Ferreira, J. A., Coelho, A., \& Weersma, L. A. (2019). The mediating effect of strategic orientation, innovation capabilities and managerial capabilities among exploration and exploitation, competitive advantage and firm's performance. Contaduria y Administracion, 64(1), 1-25. https://doi.org/10.22201/fca.24488410e.2019.1918

Becerra Rodríguez, F.; Valencia Naranjo, J. C. (2008). La innovación tecnológica en el contexto de los clúster regionales. Cuadernos de Administracion, 21(37), 133-159. http://www.scielo.org.co/pdf/cadm/ v21n37/v21n37a07.pdf

Bittencourt, B. A., Zen, A. C., \& Prévot, F. (2019). Innovation capability of clusters: Understanding the innovation of geographic business networks. Revista Brasileira de Gestao de Negocios, 21(4), 647-663. https://doi.org/10.7819/rbgn.v21i4.4016

Cabrera-Moya, D. R. (2017). Towards a framework of generating sustainable competitive advantages in the integrated public transport systems-IPTS in Colombia. Contribution of dynamic innovation capabilities | Hacia un marco de referencia para la generación de ventajas competitivas so. DYNA (Colombia), 84(200), 169-175. https://doi. org/10.15446/dyna.v84n200.54118

Cachanosky, I. (2012). Eficiencia técnica, eficiencia económica y eficiencia dinámica. Procesos de Mercado. Revista Europea de Economía Política, 9(2), 51-80. http://www.hacer.org/pdf/ICachanosky00.pdf

Cevallos, A., Duque, E., \& Echeverria, T. (2018). Validación del constructo capacidad de innovación utilizando el análisis factorial confirmatorio en Ecuador. Revista Espacios, 39(11). https://www.revistaespacios.com/a18v39n11/a18v39n11p24.pdf 
Cheng, C. C. J., \& Chen, J. S. (2013). Breakthrough innovation: The roles of dynamic innovation capabilities and open innovation activities. Journal of Business and Industrial Marketing, 28(5), 444-454. https://doi.org/10.1108/08858621311330281

Cornell University, INSEAD, \& WIPO. (2019). The Global Innovation Index 2019: Creating Healthy Lives-The Future of Medical Innovation. https://www.globalinnovationindex.org/userfiles/file/reportpdf/giifull-report-2019.pdf

Daou, A., Karuranga, E., \& Su, Z. (2013). Intellectual capital in Mexican SMEs from the perspective of the resource-based and dynamic capabilities views. Journal of Applied Business Research, 29(6), 16731688. https://doi.org/https://doi.org/10.19030/jabr.v29i6.8206

Delgado, M. de J., \& Álvarez, I. (2005). Evaluación de la eficiencia técnica en los países miembro de la Unión Europea. Gestión y Política Pública, 14(1), 107-128. https://www.redalyc.org/pdf/133/13314104.pdf

Díaz Pérez, C., Arechavala Vargas, R., Alarcón Ozuna, A., \& Ayala Arriaga, A. (2009). Economic competitiveness through cluster integration: The development of innovation capabilities in the mexican software industry. PICMET: Portland International Center for Management of Engineering and Technology, Proceedings, 338-345. https:// doi.org/10.1109/PICMET.2009.5262170

Dosi, G. (1992). Fuentes, métodos y efectos microeconómicos de la innovación. Revista Internacional de Economía, 22. https://www.researchgate.net/publication/28128148_Fuentes_metodos_y_efectos_ microeconomicos_de_la_innovacion

Enjolras, M., Camargo, M., \& Schmitt, C. (2020). Evaluating innovation and export capabilities of SMEs: Toward a multi-criteria decision-making methodology. Journal of Technology Management and Innovation, 15(3), 17-32. https://doi.org/10.4067/s071827242020000300017

Farrell, M. J. (1957). The Measurement of Productive Efficiency. Journal of the Royal Statistical Society. Series A (General). https://doi. org/10.2307/2343100

Figueiredo, P. N. (2014). Beyond technological catch-up: An empirical investigation of further innovative capability accumulation outcomes in latecomer firms with evidence from Brazil. Journal of Engineering and Technology Management - JET-M, 31(1), 73-102. https://doi. org/10.1016/j.jengtecman.2013.10.008

Fleury, A., Leme Fleury, M. T., \& Mendes Borini, F. (2013). The Brazilian Multinationals' Approaches to Innovation. Journal of International Management, 19(3), 260-275. https://doi.org/10.1016/j.intman.2013.03.003

Fried, H.O.; Knox Lovell, C.A.; Smidt, S. S. (2008). The Measurement of Productive Efficiency and Productivity Change (S. S. F. H.O., K. L. C.A., \& Smidt (eds.)). Oxford University Press. https://doi.org/ DOI:10.1093/acprof:oso/9780195183528.001.0001
Gonçalves Tondolo, V. A., Bitencourt, C. C., \& Tondolo, R. da R. P. (2010). Dynamic capabilities development to implement international strategy: the case of a brazilian wine company. Agroalimentaria, 16(31), 81-86. https://www.researchgate.net/publication/262633467_ Dynamic_capabilities_development_to_implement_international_ strategy_The_case_of_a_brazilian_wine_company

González Fidalgo, E. (1999). Recursos, capacidades, tecnología y eficiencia. XIII Congreso Nacional, IX Congreso Hispano-Francés, 579584. https://dialnet.unirioja.es/servlet/articulo? codigo $=565262$

Griffith, D. a, \& Harvey, M. G. (2001). Resource Perspective of Global Dynamic Capabilities. Journal of International Business Studies, 32(3), 597-606. https://www.researchgate.net/profile/Michael-Harvey-17/ publication/5222964_A_Resource_Perspective_of_Global_Dynamic_Capabilities/links/0fcfd50900b8b66391000000/A-ResourcePerspective-of-Global-Dynamic-Capabilities.pdf

Guan, J., \& Ma, N. (2003). Innovative capability and export performance of Chinese firms. Technovation, 23(9), 737-747. https://doi. org/10.1016/S0166-4972(02)00013-5

Herrmann, J. D., Sangalli, L. C., \& Teece, D. J. (2017). Dynamic Capabilities: Fostering an Innovation-Friendly Environment in Brazil. Revista de Administração de Empresas, 57(3), 283-287. https://doi. org/10.1590/s0034-759020170309

Jardon, C. M. (2018). Moderating effect of intellectual capital on innovativeness in Latin American subsistence small businesses. Knowledge Management Research and Practice, 16(1), 134-143. https://doi.org /10.1080/14778238.2018.1428069

Lema, R., Quadros, R., \& Schmitz, H. (2015). Reorganising global value chains and building innovation capabilities in Brazil and India. Research Policy, 44(7), 1376-1386. https://doi.org/10.1016/j.respol.2015.03.005

Liboni, L. B., Liboni, L. H. B., \& Cezarino, L. O. (2018). Electric utility 4.0: Trends and challenges towards process safety and environmental protection. Process Safety and Environmental Protection, 117, 593-605. https://doi.org/10.1016/j.psep.2018.05.027

López-Cabarcos, M. Á., Göttling-Oliveira-Monteiro, S., \& Vázquez-Rodríguez, P. (2015). Organizational Capabilities and Profitability: The Mediating Role of Business Strategy. SAGE Open, 5(4). https:// doi.org/10.1177/2158244015616852

López M., O. H., Villegas, G. C., \& Catica, J. R. (2018). Validez del instrumento para determinar los detonantes de las capacidades de innovación en las organizaciones dedicadas al cuidado de la salud. Gaceta Medica de Caracas, 126(2), 160-169. https://investigaciones-pure.udem.edu.co/es/publications/validez-del-instrumento-para-determinar-los-detonantes-de-las-cap

López Montoya, O. H., \& Acosta-Prado, J. C. (2019). Relación entre la gestión del conocimiento y la capacidad de innovacion en instituciones de salud (Colombia). Espacios, 40(28), 11. https://www.revistaespacios.com/a19v40n28/19402811.html 
Maldonado-Guzmán, G., Garza-Reyes, J. A., Pinzón-Castro, S. Y., \& Kumar, V. (2019). Innovation capabilities and performance: are they truly linked in SMEs? International Journal of Innovation Science, 11(1), 48-62. https://doi.org/10.1108/IJIS-12-2017-0139

Martínez Marín, S. J., \& Arango Aramburo, S. (2017). Dynamics simulation model for evaluating the investment in technological innovation in the capabilities of Colombia Software industries. Espacios, 38(9), 3. https://www.researchgate.net/publication/316696657_Dynamics_simulation_model_for_evaluating_the_investment_in_technological_ innovation_in_the_capabilities_of_Colombia_Software_industries

Mejía Vallejo, A., \& Arias-Pérez, J. (2017). Approach to differences in product and process innovation capabilities and financial performance in manufacturing companies. Espacios, 38(4), 11. https://www. revistaespacios.com/a17v38n04/a17v38n04p12.pdf

Moher, D., Liberati, A., Tetzlaff, J., Altman, D., \& Group, T. P. (2009). Preferred Reporting Items for Systematic Reviews and Meta-Analyses: The PRISMA Statement. PLOS Medicine, 6(7). https://journals.plos. org/plosmedicine/article?id=10.1371/journal.pmed.1000097\&utm_ medium $=$ email\&utm_source $=$ transaction

Narcizo, R. B., Canen, A. G., \& Tammela, I. (2013). SME's innovation capability as a resource to meet future logistical demands of Brazilian oil industry. Annals of Faculty Engineering Hunedoara - International Journal Of Engineering, 157-162. https://www.researchgate.net/ publication/276294075_SME's_innovation_capability_as_a_resource_to_meet_future_logistical_demands_of_Brazilian_oil_industry

Novick, M., Rotondo, S., \& Breard, G. (2013). ICT adoption, capabilities development and innovation processes in Argentina: An employment approach. African Journal of Science, Technology, Innovation and Development, 5(6), 519-529. https://doi.org/10.1080/20421338.2013.826777

OCDE, \& Eurostat. (2018). Oslo Manual 2018 (OCDE (ed.); 4th ed.). OCDE. https://doi.org/10.1787/9789264304604-en

Oliveira, C. A. O., Ruffoni, E. P., Maçada, A. C. G., \& Padula, Â. D. (2019). Innovation capabilities in the food processing industry in Brazil. British Food Journal, 121(11), 2901-2918. https://doi.org/10.1108/ BFJ-10-2018-0647

Peretto, C. B. (2017). Métodos Para Medir Y Evaluar La Eficiencia De Unidades Productivas. Revista de La Escuela de Perfeccionamiento En Investigación Operativa, 24(39), 5-25. https://revistas.unc.edu.ar/ index.php/epio/article/view/16540

Quintero S., I. C., Galvis G., A. C., Rentería T., J., \& Giraldo H., E. (2019). Capacidades de innovación. Retos y oportunidades para las microempresas. https://redipe.org/editorial/capacidades-de-innovacion/

Rajapathirana, R. P. J., \& Hui, Y. (2018). Relationship between innovation capability, innovation type, and firm performance. Journal of Innovation \& Knowledge, 3, 44-55. https://doi.org/https://doi. org/10.1016/j.jik.2017.06.002
Ramirez, S. Q., Sánchez, B. M., Jimenez, S. C., Castañeda, W. R., \& Ramirez, D. G. (2019). Avocado and Coffee Supply Chains Specialization in Colombia. Procedia Computer Science, 158, 573-581. https:// doi.org/10.1016/j.procs.2019.09.091

Robledo Velasquez, J. (2016). Introducción a la gestión de la tecnología y la innovación. In Universidad Nacional de Colombia. https://minas.medellin.unal.edu.co/descargas/Robledo_2017_Introduccion_a_ la_gestion_de_la_tecnologia_y_la_innovacion.pdf

Rodriguez, C. M., Wise, J. A., \& Martinez, C. R. (2013). Strategic capabilities in exporting: An examination of the performance of Mexican firms. Management Decision, 51(8), 1643-1663. https://doi. org/10.1108/MD-10-2012-0766

Schwab, K. (2019). Insight Report - World Economic Forum.

Serrano García, J., \& Robledo Velásquez, J. (2013). Methodology for evaluating Innovation Capabilities at university institutions using a fuzzy system. Journal of Technology Management and Innovation, 8(SPL. ISS.3), 246-259. https://doi.org/10.4067/s0718-27242013000300051

Souza, A. A. A., Alves, M. F. R., Macini, N., Cezarino, L. O., \& Liboni, L. B. (2017). Resilience for sustainability as an eco-capability. International Journal of Climate Change Strategies and Management, 9(5), 581-599. https://doi.org/10.1108/IJCCSM-09-2016-0144

Tarraço, E. L., Bernardes, R. C., Borini, F. M., \& Rossetto, D. E. (2019). Innovation capabilities for global R\&D projects in subsidiaries. European Journal of Innovation Management, 22(4), 639-659. https://doi. org/10.1108/EJIM-08-2018-0185

Teece, D. J. (2014). The Foundations of Enterprise Performance: Dynamic and Ordinary Capabilities in an (Economic) Theory of Firms. Academy of Management Perspectives, 28(4), 328-352. https://doi. org/10.5465/amp.2013.0116

Teece, D. J. (2017). A capability theory of the firm: an economics and (Strategic) management perspective. New Zealand Economic Papers, 53(1), 1-43. https://doi.org/10.1080/00779954.2017.1371208

Teece, D. J., Pisano, G., \& Shuen, A. (1990). Firm capabilities, resources and the concept of strategy. Economic Analysis and Policy Working Paper EAP 38, University of California.

Valdez-Juárez, L. E., García-Pérez-de-Lema, D., \& Maldonado-Guzmán, G. (2018). ICT and KM, Drivers of Innovation and Profitability in SMEs. Journal of Information \& Knowledge Management, 17(01), 1850007. https://doi.org/10.1142/s0219649218500077

WIPO. (2019). The Global Innovation Index 2019: Creating Healthy Lives-The Future of Medical Innovation. https://www.wipo.int/ publications/es/details.jsp?id=4434

World Economic Forum. (2018). The Global Competitiveness Report 2018. In World Economic Forum Reports 2018. https://doi.org/ISBN13: 978-92-95044-73-9 
World Economic Forum. (2019). The Global Competitiveness Report 2019. http://www3.weforum.org/docs/WEF_TheGlobalCompetitivenessReport2019.pdf

Wu, R. (2019). Firm Development and Bribery: An Empirical Study from Latin America. Atlantic Economic Journal, 1-22. https://doi. org/10.1007/s11293-019-09609-6

Yam, R. C. M., Guan, J. C., Pun, K. F., \& Tang, E. P. Y. (2004). An audit of technological innovation capabilities in Chinese firms: Some empirical findings in Beijing, China. Research Policy, 33(8), 1123-1140. https://doi.org/10.1016/j.respol.2004.05.004
Zamora Torres, A. I., \& Tello, A. F. (2018). Medición de la eficiencia de la Innovación 2013-2016 mediante el Análisis Envolvente de Datos (AED) en red dinámica. Economía, Sociedad y Territorio, 18(57), 577-584. https://doi.org/https://doi.org/10.22136/est20181184

Zartha Sossa, J. W., Rubiano Gonzalez, J. A., Estrada Reveiz, R., Guarnizo Gómez, C. A., Díaz Uribe, J. H., \& Gómez Garcés, J. (2016). Capacidades de innovación. Medición de capacidades de innovación en 460 empresas de Quindío - Colombia. Espacios, 37(10). https://www. revistaespacios.com/a16v37n10/16371002.html 